Kaleidoscope

\title{
Kochen für Kinder mit Nahrungsmittelallergien
}

- Da Grundnahrungsmittel zu den Hauptauslösern von frühkindlichen Nahrungsmittelallergien zählen, sind die diätetischen Einschränkungen für Betroffene erheblich. Bereits bei ein oder zwei klinisch relevanten Allergien auf Grundnahrungsmittel ist das Alltagsmanagement für die betroffenen Familien und das Umfeld des Kindes nicht immer leicht. Je mehr Allergien vorliegen, desto bedeutsamer ist der Stellenwert einer diätetischen Führung. In der Ernährungstherapie werden drei wichtige Ziele verfolgt: die Vermittlung von Wissen für die notwendige Meidung, die Sicherung der Bedarfsdeckung und der Erhalt der Lebensqualität.

Die beiden Autorinnen, Christiane und Birgit Schäfer, haben mit ihrem Buch "Rezepte ohne Milch, Ei, Weizen und Soja für Kinder" auf gelungene Weise demonstriert, dass die Lebensqualität auch bei Allergien auf vier wichtige Grundnahrungsmittel nicht leiden muss. Mit ihren attraktiven Rezepten für das Säuglingsalter und die ersten Kinderjahre vermitteln sie dem Leser, dass trotz umfangreicher
Meidung eine optimale Bedarfsdeckung und eine hohe Lebensqualität möglich ist.

Die Rezepte orientieren sich an den bekannten Empfehlungen des Forschungsinstituts für Kinderernährung in Dortmund, sind aber so modifiziert, dass die bestmögliche Nährstoffzufuhr erfolgt. Auf diese bewusste Abwandlung der Rezepte hätte nachdrücklich hingewiesen werden sollen, um zu verdeutlichen, dass bei steigendem Lebensalter die Spezialnahrungen als Milchersatz allein nicht ausreichen.

Als verwirrend wird die - offenbar durch den Verlag vorgegebene - Einreihung der Rezepte in die grobe Alterseinteilung 5. bis 12 . Monat empfunden. Beim flüchtigen Lesen entsteht so der Eindruck, als spielten Rezepte für Frühstück und Zwischenmahlzeiten bereits am Ende des ersten Lebenshalbjahres eine Rolle.

Sehr zu begrüßen ist dagegen die Idee der Autorinnen, die Einsatzmöglichkeiten der verschiedenen Ei-Ersatz-Alternativen übersichtlich und mit Blick auf die Küchenpraxis aufzulisten. Allein dafür lohnt sich der Kauf des Buches - übrigens auch, wenn das Kind „nur" eine Hühnereiweißallergie hat. In einem solchen Fall sollten sich die Eltern dann allerdings darüber im Klaren sein, dass sie für ihren Nachwuchs die Karenzmaßnahmen hinsichtlich Milch, Soja und Weizen nicht umzusetzen brauchen.

Unterm Strich unterstützt dieses Buch betroffene Familien dabei, die therapeutischen Maßnahmen umzusetzen. Damit festigt es die Inhalte der ernährungstherapeutischen Beratung auf vorbildliche Weise. Dr. Imke Reese

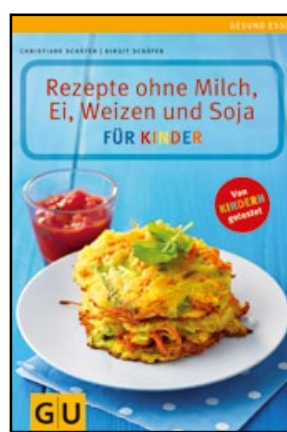

Christiane Schäfer, Birgit Schäfer Rezepte ohne Milch, $\mathrm{Ei}$, Weizen und Soja für Kinder

GU Verlag, München 2011

ISBN: 978-3-8338-

2313-8

$14,99 €$ 